\title{
e-Migrinter
}

$14 \mid 2016$

L'ajustement méthodologique comme fabrique critique du savoir dans les études migratoires

Méthodologie d'une étude en recherche biographique portant sur les « migrations précaires »

\section{Mike Gadras}

\section{(2) OpenEdition}

\section{Journals}

Édition électronique

URL : https://journals.openedition.org/e-migrinter/726

DOI : 10.4000/e-migrinter.726

ISSN : 1961-9685

Éditeur

UMR 7301 - Migrinter

Référence électronique

Mike Gadras, « Méthodologie d'une étude en recherche biographique portant sur les « migrations précaires » », e-Migrinter [En ligne], 14 | 2016, mis en ligne le, consulté le 20 mai 2021. URL : http:// journals.openedition.org/e-migrinter/726 ; DOI : https://doi.org/10.4000/e-migrinter.726

Ce document a été généré automatiquement le 20 mai 2021.

Tous droits réservés 


\title{
Méthodologie d'une étude en recherche biographique portant sur les « migrations précaires »
}

\author{
Mike Gadras
}

\section{NOTE DE L'AUTEUR}

Je souhaite vivement remercier Christine Delory-Momberger (Professeure en sciences de l'éducation, membre du Centre de recherche interuniversitaire EXPERICE, Paris 13Paris 8) pour de la confiance qu'elle me témoigne dans la direction de cette thèse.

1 Cette contribution a pour objectif de présenter les outils méthodologiques de ma recherche de thèse qui porte sur la compréhension des modes d'appréhension de l'expérience migratoire depuis le point de vue des «migrants» eux-mêmes. L'orientation de cette démarche d'étude est herméneutique ${ }^{1}$ et se donne pour champ d'investigation les processus de subjectivation des individus. Le phénomène étudié n'est donc pas tant l'expérience migratoire, en tant que telle, que les modes d'appréhension du vécu à partir desquels l'expérience vive prend forme et engendre les réalités singulières sur lesquelles se fondent l'existence et la vie quotidienne des participants de la recherche. Dans sa visée anthropologique, cette étude cherche à saisir les rapports formatifs entre les modes d'appréhension du vécu et la mise en discours du vécu d'expériences. Le développement qui suit a donc pour objet de présenter le cadre paradigmatique de la recherche et sa problématique, puis, le processus de recueil des entretiens de recherche biographique, et enfin, de présenter un procédé herméneutique s'orientant vers l'interprétation de mon corpus d'entretiens. Sur ce dernier plan, le présent article fait état d'un travail en cours d'élaboration. 


\section{Cadre épistémologique et problématique de la recherche}

«Il serait erroné de croire que tout ce qui est pensé sur le mode du flou et de l'imprécision (relatifs) soit dénué de sens ou vide de signification. Ce qui n'est vécu et conçu qu'assez vaguement est également porteur de sens. Ceci est vrai autant pour le sujet qui le vit et le pense que pour celui qui est en relation avec lui, que ce soit en tant qu'observateur ou que partenaire. Cela étant, la signification s'établit sur le mode du flou et de l'imprécision. Atteindre une clarté et une précision optimales nécessiterait une clarification au moyen d'une explication interprétative » (Schutz, 2009, p. 141).

2 Il est certes important de me rappeler, en tant que chercheur, la place et le pouvoir " performatif ${ }^{2}$ » qu'occupe le sens attribué par les hommes à leur vécu. En ce qu'ils en disent et en espèrent au présent, chaque discours est une manière de dire le monde, de le faire exister et de probablement le faire advenir. Le travail d'élucidation que chaque individu réalise pour interpréter sa propre existence ainsi que ses situations de vie en leur conférant un sens singulier, pose la condition même "d'être-au-monde ", sous les auspices de l'anthropologie philosophique et herméneutique. À ce titre, comment s'élaborent alors les réalités sociales pour les individus ? Comment conçoivent-ils, au cours de ce processus d'élaboration, leur rôle et leur place propres?

Réaliser une étude portant sur des contextes, des situations, des périodes ou des phénomènes différents ayant trait aux expériences migratoires de l'homme, ce n'est pas simplement porter un intérêt particulier à des conditions générales ou singulières de réalisation et d'effectuation de l'existence humaine. C'est également s'intéresser, parfois à son insu, aux processus à partir desquels l'homme fait de sa vie et des réalités qui la constituent une histoire singulière. Ces réalités ne sont pas que des histoires personnelles, - histoires de vie et récits d'expériences -, elles sont les traces de l'appartenance de l'homme à une "historicité »: au travers des canevas et des liens qu'il tisse avec celle-ci, il interprète son vécu d'expériences et élabore les significations de son propre parcours de vie (Delory-Momberger, 2002, 2005, 2009). Il faut entendre par « historicité », les évènements historiques, les récits populaires, les mythes et les croyances, etc., c'est-à-dire les matrices où s'élaborent, pour les hommes et les femmes, les fondements communs d'une même société.

Ma recherche doctorale a comme cadre disciplinaire les sciences de l'éducation et se déploie depuis la perspective compréhensive de l'anthropologie sociale. Menée dans le champ de la recherche biographique en éducation, cette étude vise à comprendre les modes de constitution anthropologiques de plusieurs « migrants » à partir de l'étude de récits, de leurs parcours de vie et de leurs cadres d'existence (Delory-Momberger, 2004, 2006). Le recours aux termes de «biographie» et de "biographique» ne vise pas à désigner une réalité factuelle, en ce qu'elle prétend à l'objectivité. Ce néologisme plutôt à rendre compte $\mathrm{du}$ « champ de représentations et de constructions selon lesquelles les êtres humains perçoivent leur existence » (Delory-Momberger 2009, p. 76) autant qu'au fait que la «compréhension narrative de l'expérience [renvoie] à une écriture, c'est-àdire à un mode d'appréhension et d'interprétation du vécu ayant sa dynamique et sa syntaxe, ses motifs et ses figures » (Ibid., p. 76). Située à l'intersection de ce que l'on vit et de ce que l'on en dit, la biographie n'est pour ainsi dire pas la vraie vie ; elle est, en tant que l'idée que l'on se fait de sa vie, le processus conscientisé par lequel l'individu existe et peut entrer en relation avec le monde (Delory-Momberger, 2004). 
5 Sur un plan empirique, ma recherche de «terrain » se réalise depuis trois ans auprès du groupe des Sorins ${ }^{3}$, à Montreuil, en région parisienne. Constitué en février 2011 suite à la fermeture par la police d'un «squat d'habitation » (Bouillon, 2011) où 400 personnes avaient trouvé refuge, le groupe de Sorins compte aujourd'hui 173 «migrants », tous originaires d'Afrique subsaharienne. Depuis cinq ans, ces derniers vivent dans les murs d'une ancienne usine de production où une cuisine et des dortoirs collectifs ont été aménagés. Dans un espace commun que beaucoup d'entre eux désignent comme «la cour» ou "le salon» se déroulent de nombreuses activités telles que la couture, l'épicerie au détail, les soirées football devant la télévision, le cirage de chaussures, ainsi que les soirées dansantes du vendredi soir. Cette grande diversité témoigne de la richesse de l'économie sociale qui se réalise dans cet espace commun. Malgré leurs conditions précaires d'habitation, les frontières entre lieu et non-lieu (Augé, 1992) sont souvent difficiles à délimiter, pour le chercheur. "Un espace qui ne peut se définir ni comme identitaire, ni comme relationnel, ni comme historique définira un non-lieu » (Augé, 1992, p. 100); pour l'auteur, le lieu anthropologique étant la définition symétriquement opposée de cette assertion. En effet, l'ancien local désaffecté où loge le groupe des "Sorins » se trouve être un lieu de marginalisation spatiale et sociale et, dans le même temps, un lieu de circulation et d'attente où s'organise la vie sociale du groupe avec ses codes, ses règles et ses rythmes: le "squat» est donc un lieu d'habitation d'une " communauté de l'instant », pour reprendre ici la formule d'Agier (2013, pp. 11-28).

6 Ces hommes affirment poursuivre ensemble un même objectif : obtenir une carte de séjour, une étape majeure pour atteindre le commun des vies ordinaires (Leblanc, 2010). Constitué en association depuis 2012, le Collectif des Sorins cherche à résoudre, avec le soutien d'acteurs locaux et d'habitants de la ville, les problématiques pratiques se rapportant au fonctionnement et à l'organisation de la vie collective; il s'agit aussi de faire reconnaître leur existence par le seul fait de leur présence, par leur participation à la vie locale, et les liens ainsi créés avec les habitants de leur commune d'accueil. 
Photographie $n^{\circ} 1$ : La cour intérieure, du côté douches, de l'habitat du groupe des Sorins (Montreuil, France).

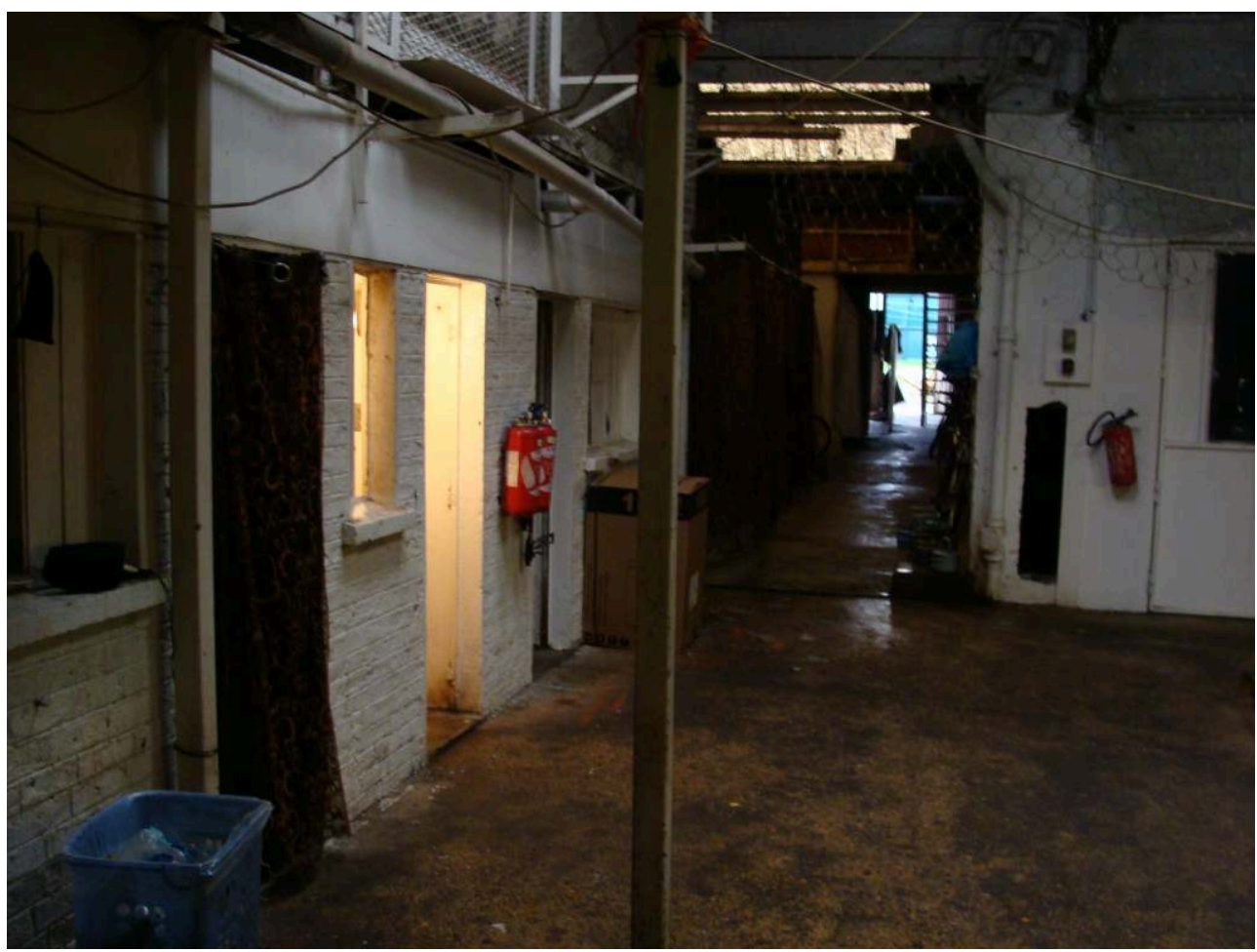

Source : Gadras, 2014

Photographie $n^{\circ} 2$ : La cour intérieure, du côté de l'espace commun, de l'habitat du groupe des Sorins (Montreuil, France)

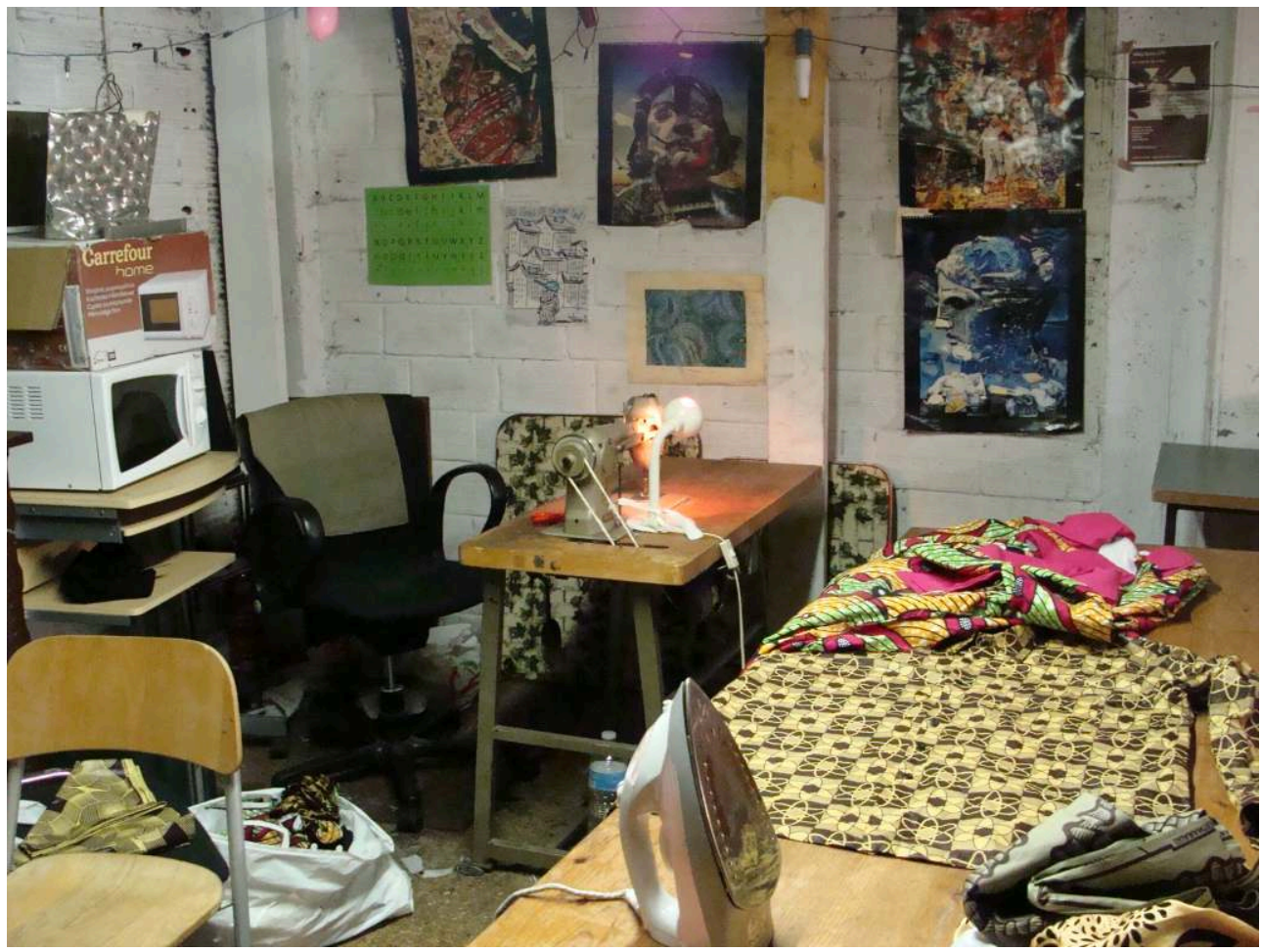

Source : Gadras, 2014 
7 Ma recherche s'oriente vers les modes de compréhension et d'interprétation de l'expérience migratoire des membres du Collectif des Sorins dont la plupart sont en situation irrégulière. C'est donc le point de vue des participants de la recherche qui est prioritairement étudié. Ainsi cette étude vise à saisir les ressorts des champs de représentation à partir desquels se configure le vécu de l'expérience et les significations qui en résultent, notamment au travers des situations de vie et des activités quotidiennes de ces « migrants ».

8 Menant ma recherche selon un procédé itératif combinant à la fois un travail de réflexions théoriques et d'observations empiriques, ma problématique de recherche s'est retrouvée petit à petit transformée. L'intentionnalité de la recherche s'articulait alors autour des tactiques et des « arts de faire » (de Certeau, 1991) mis en œuvre par les «migrants" pour vivre au quotidien et aussi autour des champs expérientiels et d'apprentissages biographiques (Alheit, Dausien, 2000; Delory-Momberger, 2008) auxquels renvoie cette forme singulière d'expérience de la société, que peut être l'expérience migratoire. Cette entreprise d'investigation, que du reste je ne néglige pas complètement dans le cadre de l'élaboration de ma recherche, s'est progressivement redéployée à partir d'une interrogation aussi simple qu'essentielle : que se passe-t-il pour ces gens? Il n'est pas possible de répondre a priori à une telle interrogation sans se heurter à de nombreuses difficultés d'ordres épistémologique, théorique et éthique. D'une certaine façon la question se pose en ces termes: qu'est-ce que vivre en "situation irrégulière» peut signifier pour un individu? Comment un individu élabore-t-il alors le sens de ce qu'il dit et fait, avec et pour son existence ? Comment rendre compte d'un tel phénomène?

9 C'est à partir de mon désir de connaître et de comprendre ces hommes que le travail ethnographique jusqu'alors envisagé s'est orienté vers des problématiques connues de la philosophie herméneutique avec laquelle la recherche biographique entretient des liens étroits. Par ce basculement de perspective, je retrouve le problème de l'« être " qui, en tant que mode de compréhension (Heidegger, Vezin, 1986), induit des manières d'habiter le monde (Delory-Momberger, 2014). En conséquence, mon investigation consiste à comprendre comment les participants à la recherche élaborent les significations qu'ils prêtent à leurs situations de vie tout comme à leur existence. Pour ce faire, je m'emploie à réaliser des entretiens de recherche biographique (DeloryMomberger, 2009) et je procède ensuite à leur examen.

10 L'analyse de ces récits a pour objectif principal d'appréhender les «attaches biographiques » qui participe à l'élaboration du sens de l'expérience dans les procès de mise en forme du vécu. Par "attaches biographiques", nous entendons signifier les repères où s'enracinent, pour l'individu, les ressorts sous-jacents aux significations qu'il prête à sa propre existence et à partir desquelles il reconnaît (et détermine) sa place, son rôle et ses activités au sein du monde social. Il s'agit dans cette perspective de réaliser une anthropologie herméneutique $d u$ "monde proposé par le texte » du récit d'expériences (Gadamer, 1999, pp. 29-55) en cherchant à examiner les différentes dynamiques de signification qui coexistent dans les réalités dont l'individu vient à rendre compte au travers de ses récits d'expériences.

11 Ce que l'on pense, ce que l'on croit, ce que l'on envisage sont autant de modalités de représentations du monde. Ces dernières tendent à produire des effets sur la conscience de l'individu et à induire de nouveaux comportements. Le concept de "performativité biographique», compris dans la perspective de Wulf (2007), vise à 
éclairer la relation anthropologique qui s'opère chez un individu, depuis son univers de sens et de représentations, entre le "penser » et l'«agir ». L'idée de performativité permet d'interroger les paradoxes, les conflictualités et les concordances, entre ce qu'un individu croit et tient pour vrai et ce qu'il fait et estime a priori adéquat. Dans ce cadre paradigmatique, ce mouvement réflexif et intentionnel est appelé « biographisation » (Delory-Momberger, 2003, 2009). Ce concept vise à décrire le travail d'organisation, d'agencement et d'orientation du vécu d'expériences de vie. C'est-à-dire qu'il permet au sujet en tant qu'« herméneutique pratique», - compris au sens de Dilthey ${ }^{4}$ (1992) -, de produire, en quelque sorte, un état de connaissances de lui-même (par réinvention et accommodation) de ses aspirations propres, tout en redéployant le monde en tant qu'horizon de possibilités. Les significations et les aspirations à partir desquelles l'homme met en représentation son existence par le biais de ses discours sur sa propre expérience du monde (et du récit qu'il en fait pour lui-même) constituent les ressorts essentiels de ce mouvement performatif. Dans un rapport pratique, ce geste de l'esprit renvoie aux possibilités d'« être » et de "faire » dont parle Sen (1992). Pour ce philosophe et économiste, vivre en tant qu' état $»^{5}$ de fait renvoie l'individu au pouvoir d' "être » et de "faire" selon la manière qu'il estime adéquate. La bonne manière est un « désir raisonné » (Ibid., p. 57) subordonné à la capacité de l'individu à déterminer par lui-même ce qui lui convient.

Si le principal effort d'élaboration de cette thèse repose sur l'analyse des récits d'expériences, les investigations qui ont précédé à la réalisation de mes entretiens constituent également une étape importante de la recherche. Cette phase critique de l'investigation met au travail et à l'épreuve la réflexivité du chercheur; elle l'oblige à s'interroger et à réajuster constamment son attitude.

\section{De l'épreuve du « terrain » aux entretiens}

Chaque vendredi soir le groupe des Sorins organise une soirée dansante à laquelle sont conviés tous les habitants de leur quartier ainsi que toutes les personnes désireuses de venir à leur rencontre. C'est en saisissant cette opportunité que je suis entré régulièrement en relation avec eux et que j'ai pu débuter mon travail d'investigation.

Dans un premier temps, il s'agit de réaliser des observations, puis de prendre appui sur des modalités de recherche se rapportant davantage à de l'observation participante et à des conversations de terrain (Becker, 1970) qui ont notamment pour but d'apprendre les codes et les règles de relations sociales se rapportant au " terrain », pour finalement aboutir à la réalisation d'entretiens de recherche biographiques. Durant mes deux premières années d'investigation, j'ai été confronté à un ensemble de contraintes que j'ai cherché systématiquement à appréhender comme des opportunités nouvelles de mieux connaître ce «terrain». Cela m'a également permis d'appréhender les conditions de vie depuis lesquelles se déploie le quotidien de ces personnes. Voici trois exemples de contraintes constantes et significatives :

a) Les premiers temps de la recherche sont marqués par l'impossibilité de dialoguer avec tous les membres du Collectif, mes échanges se limitant à quelques interlocuteurs : le porte-parole du Collectif et les membres du conseil d'administration. Les autres habitants du lieu de vie, après quelques échanges anodins, me renvoyaient systématiquement à ces derniers. Mes interlocuteurs étaient donc peu nombreux, et désignés par l'organisation du groupe. Les propos tenus par les membres « officiels » du 
Collectif ressemblaient à des mises en discours surveillés et préparés visant à produire une image maitrisée de celui-ci.

b) Les différents rythmes de vie des membres du groupe les rendent aussi peu disponibles qu'accessibles. Ils doivent régulièrement combiner les activités nécessaires à la préservation de leur propre vie avec les actions politiques du groupe. De fait, dans les rares moments de tranquillité dont ils disposent, ils ne recherchent pas la compagnie du chercheur.

c) Le Collectif regroupe actuellement 173 membres. Bien que spacieux, l'habitat est en réalité surpeuplé. L'espace individuel est particulièrement restreint et se limite dans de nombreux cas à une couchette, ce qui exclut la possibilité d'une démarche d'immersion pour le chercheur ${ }^{6}$. Il est également difficile d'avoir des échanges suivis avec des membres du groupe, ces derniers s'interpellent constamment et la promiscuité ne favorise pas la possibilité d'avoir des conversations privées et approfondies.

Photographie $n^{\circ} 3$ : Prise de vue de la mezzanine du grand dortoir montrant des toiles tendues afin de préserver autant que possible l'intimité des occupants de cet habitat (Montreuil, France)

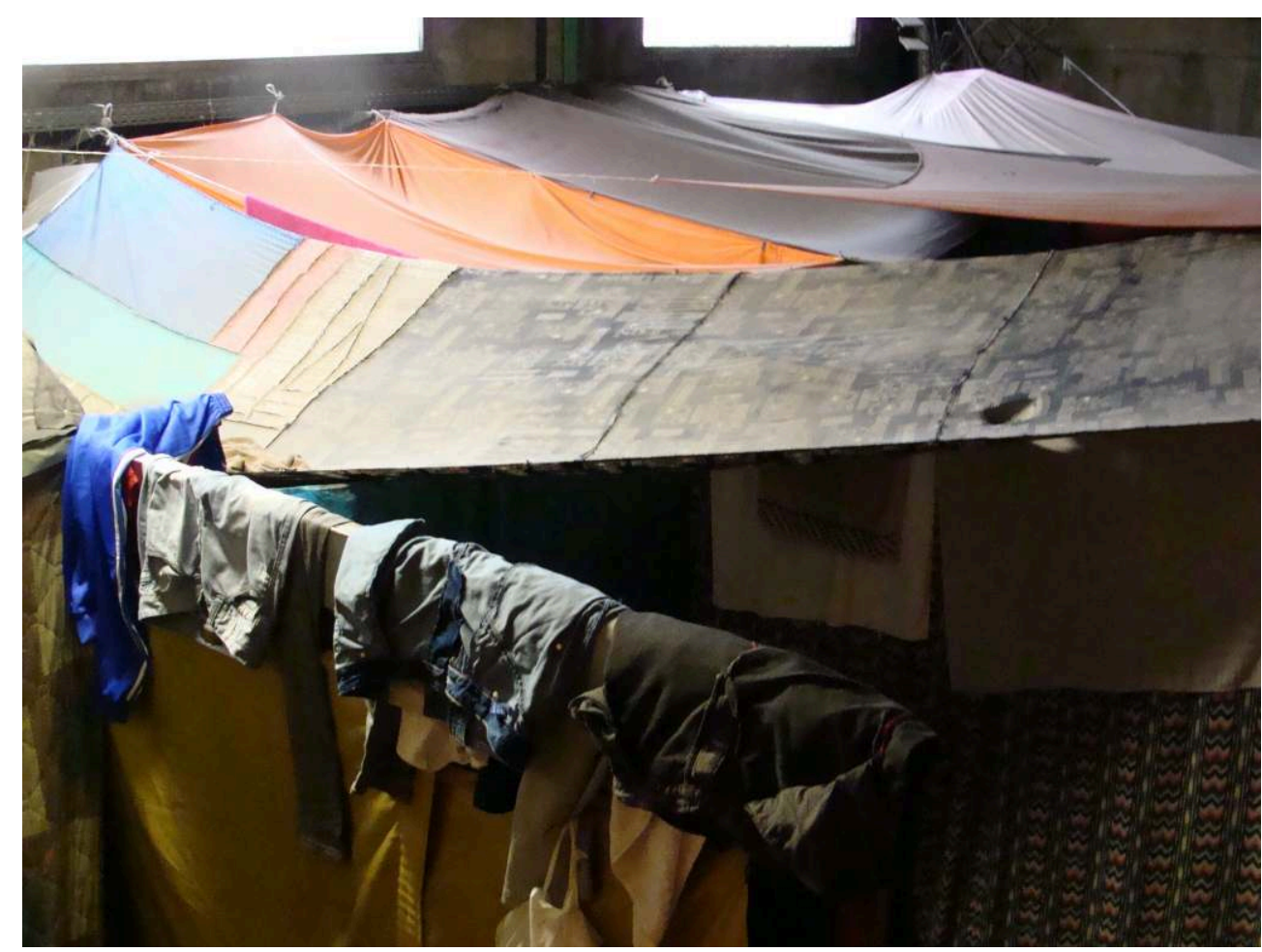

Source : Gadras, 2014 
Photographie $n^{\circ} 4$ : Un des espaces du grand dortoir de l'habitat du groupe des Sorins (Montreuil, France)

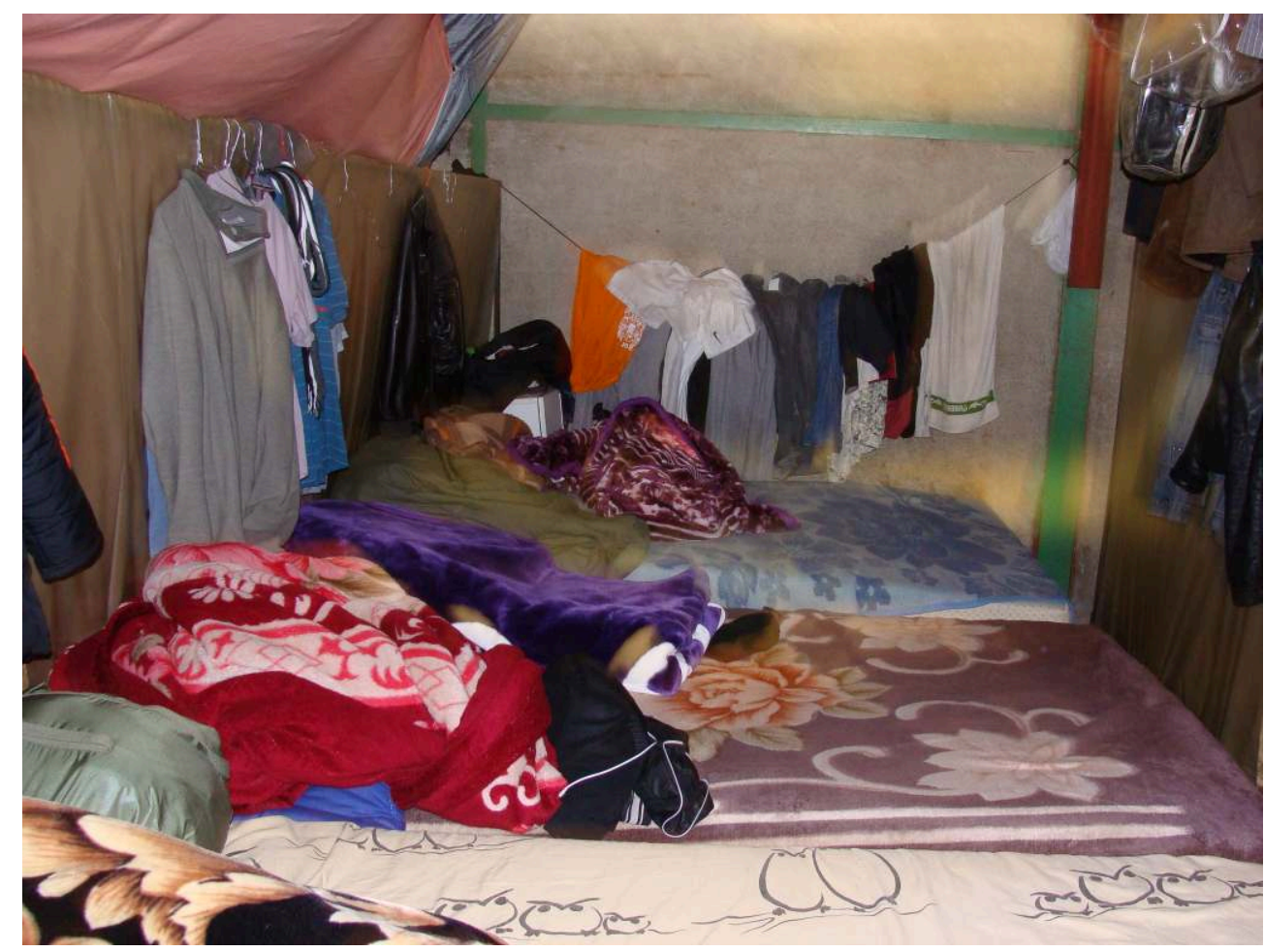

Source : Gadras, $2014^{7}$.

d) Certains membres du Collectif ont été à de nombreuses reprises interrogés par la police ou des journalistes. Ils se méfient donc des « interrogateurs » autant que de leurs propres réponses. Les outils classiques de recueil de données les rendent méfiants visà-vis du chercheur qui doit mobiliser sa mémoire dans un premier temps et remettre à plus tard la prise de notes.

e) Les migrants sont majoritairement allophones et s'expriment entre eux dans leur langue maternelle. Pour communiquer avec le chercheur, ils parlent français avec les connaissances dont ils disposent. Les membres du groupe ont des niveaux très variables de l'usage du français et cela peut constituer un obstacle lors d'un échange plus approfondi. Dans ce contexte, dialoguer ne va pas de soi et exige que le chercheur reste attentif.

15 Le temps passé auprès d'eux, les échanges autour des projets futurs, des inquiétudes individuelles et collectives, ou encore les simples « causeries » ont, peu à peu, permis de réaliser des entretiens. Bien qu'important pour le déroulement de ma recherche, ils n'étaient cependant ni ma priorité, ni une préoccupation de chaque instant. Il me semblait déterminant de prendre d'abord le temps d'être avec les membres de ce groupe et de m'imprégner de ce «terrain ». Il s'agissait d'être dans un rapport sensible à cette altérité, d'en faire l'expérience pour pouvoir par la suite mieux l'interroger et l'interpréter. L'introduction de mes carnets de «terrain » au cours des conversations et de mon journal d'investigation ${ }^{8}$ m'a permis de développer une réflexivité sur l'expérience migratoire de ces hommes, tout comme sur ma propre expérience de recherche.

16 L'approche méthodologique de l'entretien de recherche biographique se fonde sur « la parole que le sujet tient sur lui-même » (Delory-Momberger 2009, p. 77). Le participant 
de la recherche sur qui porte l'investigation doit se sentir suffisamment à l'aise dans sa relation avec le chercheur pour que ce type d'entretien puisse s'effectuer. Il est ici question d'entrer dans « les espaces-temps singuliers que chacun configure à partir de la conjugaison de son expérience (et de l'historicité de son expérience) et des mondesde-vie, des mondes communs de penser et d'agir auxquels il participe» (DeloryMomberger 2012, p. 77). S'ajustant à cette intentionnalité, l'entretien de recherche biographique constitue un double espace heuristique à partir duquel le participant de la recherche (l'enquêté) se trouve en position « d'enquêteur sur lui-même » (Ibid., p. 79) tandis que le chercheur œuvre à créer les conditions pour « comprendre le travail de l'enquêté sur lui-même » (Ibid., p. 79). Le rôle du chercheur n'est donc pas de conduire un entretien, mais de suivre l'acteur qui effectue sa propre investigation «sans jamais le dépasser » (Ibid., p. 81). De cette manière, mes entretiens débutent généralement par un questionnement relativement vague: "comment s'organisent les choses en ce moment?» ou encore "qu'as-tu en tête en ce moment?». Mes interventions se limitent ensuite à de simples relances ayant pour but de mieux comprendre ce qui est dit, sans induire les réponses.

Mon travail de thèse porte aujourd'hui sur l'étude de récits d'expériences de sept membres du Collectif des Sorins. J'ai effectué cinq entretiens avec chacun d'entre eux à des périodes différentes de ma recherche, ce qui représente un corpus de 35 recueils. Leur examen s'appuie sur les procédés d'interprétation herméneutique de Ricoeur que j'articule, en partie, avec la proposition méthodologique de Heinz (2000).

\section{L'interprétation du corpus d'entretiens : un « bricolage » méthodologique}

Réalisée dans une perspective compréhensive, cette étude n'a pas, en tant que telle, la prétention de procéder à une généralisation de ses résultats en suivant les principes de démonstration d'un quelconque modèle logique. En effet, ce n'est pas la rationalité qui est ici en jeu dans une perspective positiviste, mais toutes les éventuelles possibilités de compréhension qu'offre une démarche herméneutique s'orientant sur l' «agir » des participants de la recherche.

19 En cherchant à repérer les « attaches biographiques » à partir de l'examen des récits du vécu d'expériences, je cherche à saisir comment s'élabore, en tant que "mode d'être ", la compréhension de soi et comment s'interprètent les situations de vie dans ce contexte singulier d'existence. Ce n'est donc pas ici la démonstration qui est en jeu (en vue d'une généralisation possible) mais les modalités de compréhension de l'interprétation de l'être et de ses effets sur l'agir humain. Il s'agit de formuler des énoncés plausibles susceptibles de rendre compte du processus de formation de la signification de l'expérience de vie, à partir de l'étude des manifestations de langage reliant le « penser » à l' " agir » (" noèse » et « noème »").

Le geste fondamental qu'effectue Ricœur dans son herméneutique de la " compréhension ", «[...] consiste à proposer un partage entre le modèle du « texte ", où se pose la question du sens, et le modèle de la lecture, dans lequel se pose la question de la référence - bien que l'ensemble de la théorie herméneutique travaille à démontrer, d'une part, que le sens du texte ne peut être pensé sans l'acte de lecture, et, d'autre part, que c'est le lecteur qui accomplit la visée référentielle du texte » (Ricoeur, 
1971-1972, cité par Frey, 2008, p. 93). Il est ici fait référence au « paradigme du texte » (Ricoeur, 1986) qui est un système théorique comprenant, dans les fondements de son épistémologie, l'idée que le texte, - ici compris comme étant, à la fois, le lieu et la condition du langage -, recouvre et intègre l'expérience humaine dans son ensemble. Cette expérience est herméneutique, au sens où elle renvoie les individus à de multiples procès d'interprétation de leur existence dans lesquels la narration occupe une fonction essentielle. Depuis ce lieu et ses formes les plus diverses de manifestation, le texte constitue, en tant que champs pluriels de représentations structurées par le langage, l'ordre général depuis lequel prend forme, s'ordonne et s'articule le récit que formule l'individu à propos de son existence propre. S'inscrivant dans le prolongement de ce raisonnement, l'auteur soutient la thèse que la lecture est, en quelque sorte, la clé de voûte de l'entreprise herméneutique, et que cette dernière n'intervient donc qu'au moment où se réalise l'application de l'œuvre, c'est-à-dire le moment où s'établit la transcendance entre le sens qui émane des structures du discours (configuration) et celui auquel renvoie son objet de référence dans la perspective de son appropriation par le lecteur (refiguration).

Si la jonction entre "sens" et "référence» est, en tant que l'acte de lire, problématique, ce n'est cependant qu'à son terme que s'assemble, d'une part, l'arc herméneutique et que se réalise, d'autre part, au travers du sens et de l'objet auquel il se rapporte, l'altérité induite par le dialogue (sujet-lecteur) et la compréhension (le mode d'être) qui en résulte. Pour l'herméneutique philosophique (comme traditionnelle), l'art de l'interprétation renvoie à trois gestes interprétatifs qui sont selon Ricoeur (2013) : les subtilités de la compréhension, les subtilités de l'explication, les subtilités de l'application, qui prises ensemble, constituent l'arc herméneutique en tant qu'elles sont des modalités complémentaires de la compréhension herméneutique. «En disant que l'objet le plus général de l'interprétation est dans le discours, nous indiquons négativement que la théorie de l'interprétation ne commence pas avec l'écriture, mais que celle-ci vient spécifier des traits de communicabilité présents en tout discours, parlé ou écrit » (Ricœur cité par Frey, 2008, p. 106). Autrement dit, lire un texte ou écouter une parole revient à entrer dans la proposition d'un monde dont, pour en comprendre "l'être-au-monde " qui en découle, il reviendra au lecteur d'en faire l'interprétation et donc la lecture (sens-référence). Prenant appui sur les développements de Ricoeur, sur un plan pratique, le travail d'interprétation s'organise, selon une démarche qui m'est propre, en quatre étapes d'investigation :

1. Dans la première phase de l'interprétation (de type structural ${ }^{10}$ ), il sera question de s'intéresser à la médiation narrative du récit, c'est-à-dire à la manière dont le narrateur donne sens à son histoire de vie, en repérant : les différentes dimensions temporelles de son expérience ; la « poétique » du récit (Ricoeur, 1975) ; et ses variations imaginatives.

2. Dans la seconde phase de l'interprétation, il sera question des opérations de "mise en ordre " permettant de faire apparaître les concordances et les discordances de la mise en intrigue du récit et relatives à son identité narrative, en repérant : a) la complétude, « l'unité de composition qui exige que l'interprétation d'une partie soit subordonnée à celle de l'ensemble » (Ricoeur, 2013, p. 80) ; b) le tout, « ce qui a un commencement, un milieu, et une fin» (Ricoeur citant Aristote, Ibid., p. 80); c) l'étendue comprise comme le contour de l'action délimitée par le temps de l'œuvre ; d) la contingence entendue comme la propriété d'un événement : « est d'une certaine façon incorporée à la nécessité ou à la probabilité du récit» (Ibid., pp. 81-82). Les deux premières phases de l'interprétation nécessitent de recourir notamment au corpus théorique de Ricoeur (1975 ; 1983-1985). 
3. La troisième phase de l'interprétation, s'inspirant de la méthode de Heinz (2000) traduite par Delory-Momberger (2009) consiste à repérer les Topoï ou motifs récurrents. Cette étape de l'interprétation a pour objet de « dégager la valeur sémantique du texte, au-delà de ses seules caractéristiques sémiotiques" (Frey, 2008, p. 97). Il s'agit de thèmes qui viennent marquer singulièrement, par leur récurrence, chaque récit. En allemand Topoï signifie le lieu : ils sont relatifs aux préoccupations du narrateur et permettent de préciser les cadres d'orientation de l'interprétation. La gestion biographique renvoie au travail de réflexion sur l'imbrication et les liens qu'entretiennent les différents Topoï entre eux.

4. La quatrième phase de l'interprétation prend appui sur «la phénoménologie de l'homme capable » (Ricoeur, 2004). Il s'agit à cette étape de l'investigation de s'intéresser aux capacités de l'individu: «je peux dire, je peux faire, je peux raconter, je peux me tenir responsable de mes actions ». Le sens relatif à l'interprétation structurale est ici mis en lien avec l'action de l'individu en tant que " pouvoir être » (sens/référence) : a) «je peux dire » s'étend «à l'aspect illocutoire de tous les énoncés (Ricoeur, 2004, p. 157) et renvoie à la capacité autoréférentielle de l'individu vis-à-vis de lui-même et d'un objet; b) «je peux faire » désigne la capacité de faire et renvoie à l'être agissant (la puissance d'agir dont il se sent capable) ainsi qu'à des opérations d'objectivation (processus de biographisation) desquelles résultent dans la mise en forme de l'expérience des motifs, des causes, des aspirations, etc. ; c) « je peux raconter » (pouvoir raconter et se raconter) renvoie à l'identité narrative avec les enjeux de sa propre reconnaissance (ipséité/mêmeté) et ceux de l'altérité.

opérations de l'explication auxquelles se livre l'herméneute s'effectue la distanciation entre les mondes du texte et ses manières de l'« habiter » (Delory-Momberger, 2014) à travers les "possibilités d'être " et de choix effectifs des individus. Les mouvements complémentaires de l'interprétation et de l'explication permettent de produire un état de connaissance sur les modalités de mise en forme de l'expérience migratoire des membres de ce corpus, et de leur condition biographique ${ }^{11}$; c'est-à-dire leur manière d'être en lien avec les espaces, les temporalités, les environnements et les significations qu'ils attribuent à leur expérience ainsi qu'à leur existence, tout en leur permettant d'agir avec et sur le monde.

Dans la perspective de cette étude, la refiguration représente l'écart qu'engendrent les modes d'appréhension et de compréhension dont les discours sont la manifestation, avec les modes du "com-prendre" de l'expérience vive retenus par le chercheur (herméneute) durant sa démarche d'étude de son corpus d'entretiens. D'une certaine manière ainsi que le préconise Ricoeur, il s'agit que le texte « retourne à la vie » (1985, p. 149) en tant que contribution susceptible de participer, selon toute vraisemblance, à la description des réalités complexes qui traversent le monde et le façonnent. Au cours de son travail, les multiples pondérations du chercheur vont lui permettre de repérer et de saisir la manière dont les participants de la recherche élaborent leurs réalités de vie « en situation irrégulière ». Dans cette démarche, l'expérience vive ne peut trouver sa pleine signification que dans les inductions et les résonnances de son altérité, c'est à dire dans la pluralité de ses rapports au monde.

\section{Conclusion}

En définissant l'expérience de vie "en situation irrégulière " comme cadre global de réflexion pour tenter de saisir les modes d'appréhension et d'élaboration de l'expérience migratoire, il semble possible de recourir aux outils tout comme aux 
concepts de diverses disciplines des sciences humaines. Je pense tout particulièrement à l'anthropologie, la psycho-sociologie et la philosophie. Si le champ de la recherche biographique en éducation permet de concilier ces différentes approches, c'est l'orientation proprement herméneutique donnée à l'ensemble de ma démarche d'investigation qui rend possible la production de connaissance à partir de multiples bifurcations. De ce point de vue, les différents choix de procédés semblent receler des potentialités heuristiques susceptibles de marquer d'autant la singularité de toute démarche de compréhension. Si d'une certaine façon les subtilités de l'interprétation sont un problème général qui se pose à l'ensemble des disciplines des sciences humaines, l'orientation donnée à la démarche de cette étude est proprement herméneutique. Cela indique d'emblée que cette recherche peut s'ouvrir, en terme de résultats, vers des interprétations susceptibles de donner lieu à une compréhension "raisonnée » du phénomène étudié, au sens où l'exégèse n'a pas pour objet d'achalander entre-elles des abstractions mais d'examiner de manière systématique chaque interprétation jusqu'aux limites plausibles de l'investigation tant sur plan théorique qu'empirique. En effet, pour comprendre l'activité humaine il est souvent nécessaire de penser l'être autant que le faire. L'analyse des procès de mise en forme du vécu d'expériences permet de tenter de saisir la manière dont l'être humain vient à dire quelque-chose de sa vie et à s'y inscrire.

À partir du cadre paradigmatique de la recherche biographique et de mon corpus d'entretiens, je peux donc étudier comment ces «migrants " incarnent au travers de leur mode d'être et de leurs formes d'inscription et de présence en société, leur " possibilité d'être » ainsi que « leur puissance de vie ». Ce travail de recherche doctoral se veut être une contribution compréhensive au processus d'élaboration du sens dans les cadres singuliers de l'expérience migratoire dite "clandestine » ou "irrégulière ». Le travail de "terrain » auprès de cette population en situation de marginalité sociale nécessite de nombreux ajustements, et ce, tant d'un point de vue méthodologique que relationnel. Ce sont donc les acteurs qui fixent le rythme de la recherche, et le chercheur doit faire preuve d'inventivité pour s'approprier les opportunités situationnelles. Le travail d'interprétation visant à mettre à jour l'œuvre anthropologique de la parole chez les acteurs de l'expérience situe le travail de recherche autant dans une attention de chaque instant que dans une réflexivité accrue. Les procédés de l'interprétation herméneutique sont une ouverture à cette altérité, au sens où, de la volonté de comprendre, résulte une attention du lecteur orientée vers l'objet (le texte) dont il veut s'approprier le sens.

\section{BIBLIOGRAPHIE}

Agier, Michel (2013) Le campement urbain comme hétérotopie et comme refuge. Vers un paysage mondial des espaces précaires, Brésil(s) sciences humaines et sociales, pp. 11-28. 
Alheit, Peter ; Dausien, Bettina (2000) Die biopgraphische Konstruktion der Wirklichkeit. Überlegungen zur Biographicität des Sozialen, in Hoerning, E. (ed.) Biographische Sozialisation, Stuutgart, Lucius \& Lucius, pp. 257-284.

Augé, Marc (1992) Non-lieux, Introduction à une anthropologie de la surmodernité, Paris, Éditions du Seuil, $150 \mathrm{p}$.

Becker, Howard (1970) Sociological Work, Chicago, Aldine, X-358 p.

Bouillon, Florence (2011) Le squat, Regards croisés sur l'économie, n 9, pp. 75-84.

De Certeau, Michel (1991) L'invention du quotidien 1. Arts de faire, Paris, Galimard, LII-349 p. (Folio essais ; 146).

Delory-Momberger, Christine (2003) Biographie et éducation. Figures de l'individu-projet, Paris, Anthropos, 133 p.

Delory-Momberger, Christine (2004) Les Histoires de vie. De l'invention de soi au projet de formation, Paris, Anthropos, 289 p.

Delory-Momberger, Christine (2005) Histoires de vie et recherche biographique en éducation, Paris, Anthropos, 177 p.

Delory-Momberger, Christine (2009) La condition biographique. Essais sur le récit de soi dans la modernité avancée, Paris, Téraèdre, 122 p.

Delory-Momberger, Christine (2012) De la recherche biographique en éducation. Fondements, méthodes, pratiques, Paris, Téraèdre, $214 \mathrm{p}$.

Frey, Daniel (2008) L'interprétation et la lecture chez Ricœur et Gadamer, Paris, PUF, 300 p.

Gadamer, Hans-Georg (1999) Herméneutique et philosophie, Paris, Bauchesne, XIX-162 p. (Le Grenier à sel).

Heidegger, Martin ; Vezin, François (trad.) (1986) Être et temps, Paris, Gallimard, 600 p. (Bibliothèque de philosophie).

Heinz, Walter (2000) Selbstsozialisation im Lebenslauf. Umrisse einer Theorie biographischen Handelns = Autosocialisation dans le cours de la vie. Esquisse d'une théorie de l'agir biographique, in Hoerning, E. M. (dir.) Biographische Sozialisation, Stuttgart, Lucius \& Lucius, pp. 165-184.

Leblanc, Guillaume (2010) Dedans, dehors. La condition de l'étranger, Paris, Seuil, 217 p.

Ricoeur, Paul (1975) La métaphore vive, Paris, Seuil, 415 p.

Ricoeur, Paul (1983) Temps et Récits, Tome I, Paris, Seuil, 315 p.

Ricoeur, Paul (1984) Temps et Récits, Tome II, Paris, Seuil, 122 p.

Ricoeur, Paul (1985) Temps et Récits, Tome III, Paris, Seuil, 216 p.

Ricoeur, Paul (1986) Du texte à l'action. Essais d'herméneutique II, Paris, Seuil, 452 p. (Point Essais).

Ricoeur, Paul (2013) Cinq études herméneutiques, Genève, Labor et Fides, 139 p.

Schutz, Albert (2009) Contribution à la sociologie de l'action, Paris, Hermann, 177 p.

Sen, Amartya (1992) Repenser l'inégalité, Paris, Seuil, 318 p.

Wulf, Christophe (2007) Une anthropologie historique et culturelle. Rituels, mimésis sociale et performativité, Paris, Téraèdre, $190 \mathrm{p}$. 


\section{NOTES}

1. L'herméneutique est l'art de comprendre et d'interpréter. Dans la perspective des travaux Paul Ricoeur (1986), l'explication et l'interprétation constituent les deux mouvements complémentaires de la compréhension. En expliquant le monde du texte, il est possible selon l'auteur de se livrer à l'interprétation de son œuvre dans l'agir humain et ainsi de l'objectiver et de le comprendre.

2. La notion de pouvoir performatif vise ici à rendre compte des effets narratifs et effectifs qu'induisent sur l'agir humain les significations qu'un individu attribue à son vécu.

3. En se constituant formellement, les membres du groupe des « Sorins » décideront de conserver le nom de l'adresse où ils auront trouvé refuge durant plusieurs mois au 94, rue des Sorins. Ainsi, le groupe porte le nom du lieu où s'initient les rencontres entre ses futurs membres.

4. Pour Dilthey (1992), le processus de construction du sens se configure à partir des expériences que réalise un individu.

5. Vivre est la forme active à laquelle se rapporte tout «état » du vivant et de la vie. Pour des philosophes tels que Sen ou Ricoeur, cet état du vivant, - en tant qu'état de fait s'opposant radicalement à la mort -, induit chez l'homme des capacités ainsi qu'une " puissance d'agir ».

6. Il conviendra également d'indiquer que le Collectif est uni par une lutte politique. Le groupe a besoin de moments de repli pour travailler, dialoguer et même pour gérer des conflits. Ces moments leurs appartiennent et la présence d'une personne extérieure n'est pas souhaitée.

7. Cet espace, comme l'ensemble des pièces de l'habitat, fait aujourd'hui l'objet de travaux de réhabilitation financés d'une part par la mairie, et d'autre part par deux opérations de crownfunding. Les travaux sont coordonnés par les architectes de l'urgence de chez Habitats Solidaires à Montreuil.

8. Le journal d'investigation est un outil réflexif. Il a pour objet de soutenir une démarche globale de recherche à toutes les étapes de son développement. Il permet d'annoter spontanément des idées et des observations se rapportant de près ou de loin à la recherche. On y trouve autant des annotations relatives à des revues de presse ou des ouvrages universitaires, ainsi que des commentaires personnels portant sur le travail de recherche.

9. Pour Husserl (1968) la « noèse » est l'acte même de la pensée alors que le « noème » est l'objet intentionnel de cette pensée en tant que objet irréel, en ce sens où il n'est pas une chose ou un aspect d'une chose préexistante.

10. L'explication structurale se fixe comme objet de dégager les caractéristiques sémiotiques du texte. Il s'agit pour Ricoeur d'aller saisir le sens déjà constitué et marqué par les structures du texte.

11. J'emprunte ici la notion de «condition biographique » au titre de l'ouvrage de DeloryMomberger (2009).

\section{INDEX}

Index géographique : France

Mots-clés : clandestins, migration irrégulière, vie quotidienne, biographie, histoire de vie, méthodologie, étude de cas 
AUTEUR

MIKE GADRAS

Doctorant en Sciences de l'Éducation, Paris 13 Sorbonne, Paris Cité (EXPERICE)

mike.gadras@gmail.com 